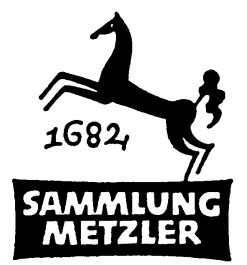

REALIEN ZUR LITERATUR

ABT. D

LITERATURGESCHICHTE 


\title{
PETER PUTZ
}

\section{Friedrich Nietzsche}

2., durchgesehene und ergänzte Auflage

\author{
MCMLXXV \\ J. B. METZLERSCHE VERLAGSBUCHHANDLUNG \\ STUTTGART
}


ISBN 978-3-476-12062-5

ISBN 978-3-476-98801-0 (eBook)

DOI 10.1007/978-3-476-98801-0

M 62

(C) 1975 Springer-Verlag GmbH Deutschland

Ursprünglich erschienen bei J.B. Metzlersche Verlagbuchhandlung und Carl Ernst Poeschel Verlag GmbH in Stuttgart 1975 


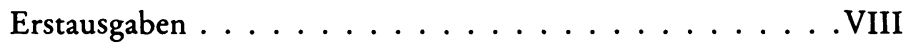

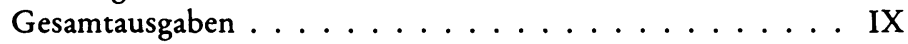

Briefe . . . . . . . . . . . . . . . . X

Bibliographien und Hilfsmittel . . . . . . . . . . X

I. LeBen $\ldots \ldots \ldots \ldots \ldots \ldots \ldots \ldots \ldots$ I

II. Philosophie . . . . . . . . . . . . . 7

I. Geschichte der Nietzsche-Deutung . . . . . . . 7

2. Der Wille zum Ganzen als Perspektivismus . . . . . 22

3. Ästhetik als Philosophie des Lebens . . . . . . . . 27

a) Ursprung und Maßstab der Kunst . . . . . . . . 27

b) Kunst und Erkenntnis. . . . . . . . . . 35

c) Der Künstler . . . . . . . . . . . . . . 37

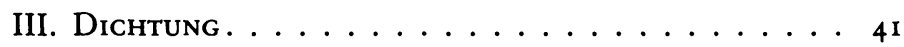

I. Prosastil. . . . . . . . . . . 4 4I

2. "Also sprach Zarathustra« . . . . . . . . . . 46

3. Lyrik . . . . . . . . . . . . . 57

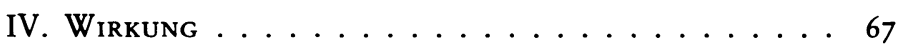

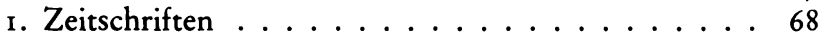

2. Mode des Ubermenschen. . . . . . . . . . . . 70

3. Naturalismus . . . . . . . . . . . . 72

4. Gegen den und neben dem Naturalismus (Spitteler, Dehmel, Schnitzler, Bahr, Hofmannsthal, Zweig, George, Kosmiker, Paul Ernst, Morgenstern, Heinrich Mann, Charon-Kreis, Rilke, Hesse, Kafka) . . . . 77

s. Expressionismus . . . . . . . . . . . 8 89

6. Thomas Mann ............... 95

7. Musil . . . . . . . . . . . . . . 100

8. Nationalsozialismus . . . . . . . . 102

9. Gegenwart . . . . . . . . . . . . . ros

10. Anhang:

a) Literatur zur Nietzsche-Wirkung im Ausland . . . 109

b) Literatur zur Nietzsche-Wirkung in der Musik . . I 17

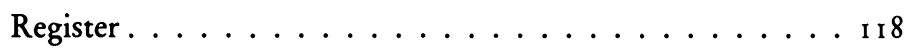


In der nachfolgenden Arbeit werden Nietzsches Werke nach der Musarion-Ausgabe zitiert. Da ein Hauptakzent auf der Wirkungsgeschichte liegt, muß auf den Text zurückgegriffen werden, von dem die Wirkung ausging. Die Musarion-Ausgabe folgt in wesentlichen Punkten ("Wille zur Macht « etc.) der Naumannschen Großoktavausgabe, die heute schwer greifbar ist. Siglen wie MA, UB etc. sind Abkürzungen für Werktitel und werden auf S. XII aufgelöst; römische Ziffern bezeichnen die Band-, arabische die Seitenzahl.

Die Literaturangaben zu Leben und Philosophie bringen nur eine sehr kleine Auswahl, die zu Dichtung und Wirkung dagegen wollen möglichst viel erfassen. Allgemeine Literatur wird am Ende eines Hauptteils, spezielle am Schluß eines Kapitels oder Abschnitts genannt. Gelegentliche Wiederholungen einzelner Titel sind dabei unvermeidbar. Innerhalb der Sachgebiete sind die Veröffentlichungen historisch nach Erscheinungsjahren geordnet. Das hat den Vorzug, daß der Leser sofort überblickt, wann über Nietzsche geschrieben wird, welche Fragen zu welcher Zeit vorherrschen, was unbeachtet bleibt usw. Schon hierdurch wird ein Stück Nietzsche-Wirkung sichtbar. Einigen Titeln folgt eine, in eckige Klammern gesetzte, charakterisierende Bemerkung des Verfassers, welche die Einführung in den großen Stoff erleichtern soll. Wichtige Thesen und Beobachtungen der Sekundärliteratur werden im Text referiert und häufig in die eigene Darstellung -eingearbeitet. Der Name des betreffenden Autors steht am Ende eines Satzes in Klammern. Er ist zuständig für den zuletzt behandelten, zusammenhängenden Gedanken. 
Kritische Stimmen zur ersten Auflage vermißten die Berücksichtigung der theologischen Nietzsche-Rezeption. Dennoch wird sie auch weiterhin ausgespart; einmal, weil sie außerordentlich diffus und daher in dem engen Rahmen nicht zu fassen ist; zum anderen, weil dieser Band in erster Linie ein Realienbuch für Germanisten sein soll. Aus demselben Grunde erhält auch die Philosophie bei weitem nicht die ihr gebührende Bedeutung.

Die zweite Auflage ergänzt die Literaturangaben um ca. 400 Titel. Sie sind Zeugnis der seit einigen Jahren merklich wachsenden Beachtung Nietzsches. Thesen und Ergebnisse wichtiger Neuerscheinungen sind im Text verarbeitet. Die Abschnitte über Wedekind und den Einfluß in England sind nur geringfügig, die Kapitel über den Nationalsozialismus und die Gegenwart dagegen erheblich erweitert worden. Neu hinzugekommen sind die Partien über Heinrich Mann, Kafka, Sternheim und über die Nietzsche-Rezeption der kritischen Theorie. 
Die Geburt der Tragödie aus dem Geiste der Musik. Leipzig: Fritzsch 1 872; 2. Aufl. Ebda 1874; Neue Ausgabe mit dem Versuch einer Selbstkritik. Leipzig: C. G. Naumann 1886 .

Unzeitgemässe Betrachtungen. Erstes Stück: David Strauss der Bekenner und Schriftsteller. Leipzig: Fritzsch 1873.

Unzeitgemässe Betrachtungen. Zweites Stück: Vom Nutzen und Nachtheil der Historie für das Leben. Ebda I 874.

Unzeitgemässe Betrachtungen. Drittes Stück: Schopenhauer als Erzieher. Ebda I 874 .

Unzeitgemässe Betrachtungen. Viertes Stück: Richard Wagner in Bayreuth. Chemnitz: Schmeitzner I 876. - Französ. Ebda 1877.

Menschliches, Allzumenschliches. Ein Buch für freie Geister. Ebda I 878. Neue Ausgabe mit einer einführenden Vorrede, 2 Bde. Leipzig: Fritzsch I 886, ${ }^{3}$ I 894 . [Enthält "Menschliches, Allzumenschliches * bis einschließlich $\approx$ Der Wanderer und sein Schatten $*$.]

Menschliches, Allzumenschliches. Ein Buch für freie Geister. Anhang: Vermischte Meinungen und Sprüche. Chemnitz: Schmeitzner I 879. - 2. Aufl. s. 0 .

Der Wanderer und sein Schatten. Ebda I880. - 2. Aufl. s. o.

Morgenröthe. Gedanken über die moralischen Vorurtheile. Ebda I88 r; Neue Ausgabe mit einer einführenden Vorrede. Leipzig: Fritzsch [dann:] C. G. Naumann I 887 .

Die fröhliche Wissenschaft. Chemnitz: Schmeitzner 1882; Neue Ausgabe mit einer Vorrede. Leipzig: Fritzsch [dann:] C. G. Naumann I 887. [Hinzugekommen sind ein 5. Buch und "Anhang: Lieder des Prinzen Vogelfrei . $^{-]}$

Also sprach Zarathustra. Ein Buch für Alle und Keinen. Chemnitz: Schmeitzner I 883; 2. Aufl. Leipzig: C. G. Naumann 1887 [mit Teil II u. III].

Also sprach Zarathustra. Ein Buch für Alle und Keinen. II. Chemnitz: Schmeitzner I883; 2. Aufl. Leipzig: C. G. Naumann I 887 [mit Teil I. u. III].

Also sprach Zarathustra. Ein Buch für Alle und Keinen. III. Chemnitz: Schmeitzner I 884; 2. Aufl. Leipzig: C. G. Naumann I 887 [mit Teil I. u. II]. Also sprach Zarathustra. Ein Buch für Alle und Keinen. [IV.] Privatdruck. Leipzig I88 5; IV. und letzter Theil. Nebst Anhang: Dionysos-Dithyramben. Leipzig: C. G. Naumann I891.

Jenseits von Gut und Böse. Vorspiel einer Philosophie der Zukunft. Ebda I 886, ${ }^{2}$ I 892 .

Zur Genealogie der Moral. Eine Streitschrift. Ebda $1887,{ }^{2} 1892$.

Der Fall Wagner. Ein Musikanten-Problem. Ebda I 888, ${ }^{2} 1892$. - Französ. Ebda 1893.

Götzen-Dämmerung, oder: Wie man mit dem Hammer philosophiert. Ebda I889. 
Dionysos-Dithyramben. Ebda I89 I [zusammen mit »Also sprach Zarathustra IV ].

Der Antichrist. Fluch auf das Christenthum. 1895 = Großoktavausg. Bd 8 [s. u.].

Nietzsche contra Wagner. Aktenstücke eines Psychologen. Privatdruck I 889; dann I 895 in Bd 8 der Großoktavausg. [s. u.].

Ecce homo. Wie man wird, was man ist. Liebhaberausgabe in beschränkter Aufl. Leipzig: Insel-Verlag 1908; dann 19 I I in Bd is der Großoktavausg. [s. u.].

Die Nietzsche-Ausgaben bis 1939 weist das «Verzeichnis der bis 1939 erschienenen Ausgaben der Werke, Kompositionen und Briefe Nietzsches* nach. In: Jahresheft für 1939 der 'Stiftung Nietzsche-Archiv«, Weimar.

\section{Wichtige Gesamtausgaben}

Großoktavausgabe: Gesamtausgabe in 19 Bden. Leipzig: C. G. Naumann I $894 \mathrm{ff}$. [Bd I-8 von Nietzsche selbst veröffentlichte Schriften; Bd 9-I6: Nachlaß; Bd 17-19: Philologica]; 2. Aufl. 1901/13. - 1926 erschien ein 20. $B d$ : Nietzsche-Register, von Richard Oehler.

Musarion-Ausgabe: Werke in 23 Bden. Hrsg. von Richard Oehler, Max Oehler u. Friedr. Chr. Würzbach. München: Musarion-Verlag 1920/29.

Kröner-Ausgabe: Werke in 12 Bden [Dünndruck]. Hrsg. v. Alfred Baeumler. Leipzig [später: Stuttgart]: A. Kröner Verlag I $930 \mathrm{ff}$.

Historisch-kritische Gesamtausgabe der Werke und Briefe. Von der Stiftung Nietzsche-Archiv veranstaltet. München: C. H. Beck 1933 ff. [Abgeschlossen nur s Bde: Werke bis zur ersten Basler Zeit $1868 / 69$, u. 4 Bde Briefe: bis 1877 .]

Werke in 3 Bden. Hrsg. v. Karl Schlechta. München: Carl Hanser 1956, ${ }^{2}$ 1960. - Als Taschenbuchausgabe bei Ullstein (s Bde., Nr. 2907-291 I). [Schlechta kann nachweisen, daß Elisabeth Förster-Nietzsche Briefe ihres Bruders an Mutter und Freunde fälschte und an sich selbst umadressierte, um ihre Person in einem günstigen Licht erscheinen zu lassen: Bd $3, \mathrm{~S}$. I 37 Iff. - Am folgenschwersten ist Schlechtas Auflösung des von den Hrsgn der Großoktavausgabe systematisierten Nachlasses: „Der Wille zur Macht. Versuch einer Umwerthung aller Werthe «. Diese Anordnung er-

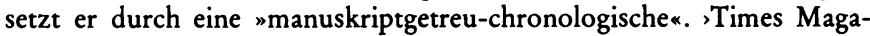
zine،, ,Les Nouvelles Littéraires‘, ,Die Welt‘ u. a. Blätter nehmen daraufhin leidenschaftlich Partei. Rudolf Pannwitz wendet sich im >Merkur،, Nov. 1957, gegen die Zerstörung des Nietzsche-Bildes; Schlechta verteidigt sich in den Frankfurter Heften«, Febr. 1958; Wolfram von den Steinen leistet Pannwitz im 'Merkur<, Aug. 1958, Schützenhilfe, und Karl Löwith kritisiert Alfred Baeumler, weil er "Die ewige Wiederkunft des Gleichen*, und Schlechta, weil er den "Willen zur Macht« vernachlässige. Der Hrsg. entgegnet den Einwänden in: Karl Schlechta "Der Fall Nietzsche«. München: Carl Hanser 1959]. 
Sämtliche Werke in 12 Bden. Neudruck der Kröner-Ausgabe [s. o.]. Stuttgart: A. Kröner Verlag 1965 . [Bd 12 bringt das Nietzsche-Register von Richard Oehler, s. u.].

Nietzsche Werke. Kritische Gesamtausgabe. Hrsg.: Giorgo Colli u. Mazzino Montinari. Berlin: de Gruyter $1967 \mathrm{ff}$. [Bisher sind in is Bdn. (III.-VIII. Abteilung) fast alle wichtigen Schriften und nachgelassenen Fragmente von der "Geburt der Tragödie " an erschienen. Die von N. selbst veröffentlichten Werke und die Nachlaßfragmente werden in streng chronologischer Reihenfolge gedruckt; das gilt auch für den Nachlaß vom Herbst 1887 bis Januar 1889 , der früher fälschlich unter dem Titel "Der Wille zur Macht " publiziert wurde.]

\section{Ausgaben Der Briefe}

Friedrich Nietzsches gesammelte Briefe. s Bde. Berlin: Schuster \& Loeffler 1900ff.; 2. Aufl. Leipzig: Insel-Verlag $1903 \mathrm{ff}$.

Briefwechsel mit Franz Overbeck. Hrsg. v. Richard Oehler u. Carl Albrecht Bernoulli. Leipzig: Insel-Verlag 1916.

Briefwechsel mit Erwin Rohde. Hrsg.v. Elisabeth Förster-Nietzsche u. Fritz Schöll. Ebda ${ }^{3} 1923$.

Briefe an Peter Gast. Hrsg. v. Peter Gast. Ebda ${ }^{3} 1924$.

Peter Gasts Briefe an Nietzsche. 2 Bde. München: Verlag der NietzscheGes. $1923 / 24$.

Briefe an Mutter und Schwester. Hrsg. v. Elisabeth Förster-Nietzsche. Leipzig: Insel-Verlag ${ }^{3} 1926,{ }^{4} 1929$.

Karl Strecker: Nietzsche und Strindberg. Mit ihrem Briefwechsel. München: Georg Müller I 92 I.

Der kranke Nietzsche. Briefe seiner Mutter an Franz Overbeck. Hrsg. v. Erich F. Podach. Wien: Bermann-Fischer 1937.

\section{Bibliographien UNd Hilfsmittel}

Friedrich Würzbach: Nietzsche. Ein Gesamtüberblick über die bisherige Nietzsche-Literatur. In: Literarische Berichte aus d. Gebiet der Philosophie. H. 19/20. Erfurt: K. Stenger 1929.

Herbert W. Reichert/Karl Schlechta: International Nietzsche Bibliography, compiled and edited. Chapel Hill/NC/USA: University of North Carolina 1960, revised and expanded 1968; fortgesetzt bis $197 \mathrm{I}$ in: N.Studien 2, 1973, S. 320-339. [Nennt über 5000 Titel in 28 Sprachen, innerhalb der Landessprachen alphabetisch geordnet.]

Richard Oebler: Nietzsche-Register. Alphabet.-systemat. Ubersicht zu Nietzsches Werken nach Begriffen, Kernsätzen u. Namen. Leipzig: A. Kröner 1926; Neudruck als Bd 12 der "Sämtl. Werke " [s. o.], ebda 1965. Musarion-Ausgabe: die Bde 22 u. 23 sind Register-Bde. 1928/29. [s. o.] Karl Schlechta: Nietzsche-Index zu den "Werken in 3 Bden «. München: Carl Hanser 1965. 
Alfred Baeumler: Nietzsche in seinen Briefen u. Berichten der Zeitgenossen. Die Lebensgeschichte in Dokumenten. Leipzig: A. Kröner 1932. [Kröners Taschenausgabe. 100.]

Karl Schlechta: Nietzsche. Zeit- und Lebenstafel. Philologischer Nachbericht. In Bd 3 seiner "Werke in 3 Bden « [s. o.], 1956, ${ }^{2} 1960$, S. I 359-1 432. Ivo Frenzel: Friedrich Nietzsche in Selbstzeugnissen und Bilddokumenten dargest. (rowohlts monographien. I I S). Reinbek: Rowohlt 1966, ${ }^{4} 1970$.

Friedrich Würzbach: Nietzsche. Sein Leben in Selbstzeugnissen, Briefen und Berichten (Goldmanns gelbe Taschenbücher Bd. 1753/54). München: Goldmann 1966.

Ernst Pfeiffer (Hrsg.): Fr. N., Paul Rée, Lou von Salomé. Dokumente ihrer Begegnung. 1970.

Richard Frank Krummel: N. u. der deutsche Geist. Ausbreitung u. Wirkung des Nietzscheschen Werkes im deutschen Sprachraum bis zum Todesjahr des Philosophen. Ein Schrifttumsverzeichnis der Jahre 1867-1900. 1974 [vgl. S. 108.] 


\section{SigLen}

EH "Ecce homo"

F "Der Fall Wagner"

FW "Die fröhliche Wissenschaft"

GM "Zur Genealogie der Moral"

GT "Die Geburt der Tragödie"

$\mathrm{H} \quad$ "Homer und die klassische Philologie"

J "Jenseits von Gut und Böse"

MA "Menschliches, Allzumenschliches «

NW "Nietzsche contra Wagner"

PW "Ueber das Pathos der Wahrheit"

UB "Unzeitgemässe Betrachtungen“

WL "Ueber Wahrheit und Lüge im aussermoralischen Sinne «

WM "Der Wille zur Macht «

$\mathrm{Z} \quad$ "Also sprach Zarathustra»

DVjs. „Deutsche Vierteljahrsschrift für Literaturwissenschaft und Geistesgeschichte «

GRM "Germanisch-Romanische Monatsschrift" Jb., Jbb. "Jahrbuch, Jahrbücher "

PMLA "Publications of the Modern Language Association of America" 